\title{
ATR-FTIR Assessment of the Biochemical Composition and Micro Hardness of the Hard Tissues of Oral Cavity Submitted to Gamma Irradiation
}

\author{
Wilber EB Paredes* and Delvonei Alves de Andrade
}

Nuclear and Energy Research Institute IPEN/CNEN Sao Paulo, Brazil

\begin{abstract}
Back ground: Clinical radiotherapy is one of the most important techniques for the treatment of malignant lesions in head and neck; however, exposure to ionizing radiation may lead to both systemic and local complications, immediately and after this treatment, where the main issue is the xerostomia and its consequent oral mucositis. Regarding late complications produced by radiation, decay of radiation and osteoradionecrosis, both dosedependent lesions, showed a high level of incidence in recent decades and it would be difficult to manage, although patients referred after treatment's completion and under the influence of local factors.
\end{abstract}

Methodology: Then, this study evaluated the effect of ionizing radiation from gamma source onto samples of enamel, root dentin and jawbone samples undergone to the same absorbed dose/dose rate that those in patients with head and neck cancer. The non-irradiated and irradiated samples were analyzed by microhardness surface analysis; scanning electron microscopy (SEM) and Fourier transform infrared spectrometry (ATR-FTIR).

Results and Discussion: Microhardness data were analyzed statistically with a confidence level of $95 \%$ ( $p$ value $<0.05 \%$ ), using parametric Student's t-test for related averages and ANOVA statistical test, finding a statistically significant result $(p=0.00)$ to all four groups samples.

Conclusion: It was observed a high deleterious and statistical significant effect of gamma radiation on hard tissues from oral cavity, regarding to physical, compositional and morphological properties.

Keywords: Osteoradionecrosis; Microhardness; Dental enamel; Root dentin; Mandibular bone; Spectroscopy; Radiotherapy

\section{Introduction}

Cancer has constituted a public health problem, so its control and prevention must prioritized in the world. Cancers develop with multiple stages over the years, so some types could avoided by eliminating exposure to the determinants and contributing factors [1]. If the malignancy potential is detected before the cells become malignant at an early stage of the disease, the treatment can be very effective and with high chances of cure [2].

Among the conventional surgical treatments commonly used in cancer treatment, other therapies, like radiotherapy and chemotherapy are widely used as definitive or adjuvant therapy for the surgical procedure, mainly in cancers of the head and neck [3]. However, high doses of radiotherapy in large areas, such as the oral cavity, upper jaw, mandible, and salivary glands may result in undesirable effects, immediate or late onset, where osteoradionecrosis (ORN) has been considered the worst [4].

ORN is one of the most severe and serious oral complications of radiation therapy for head and neck cancer. Currently literature $[3,4]$ holding that, despite the improvement in oral care performed before radiotherapy, the incidence of ORN has increased significantly in recent years almost $1 \%$ to $30 \%$ per year [3]. It has been defined as the irradiated necrotic bone exposed, which does not heal over a period of three or six months without the presence of tumor remained or tumor recurrence. This pathological entity covers both jaws, the upper jaw region and the mandibular region, with a high incidence rate in the region of the mandibular body and retro molar region documented in recent years [4-7].
The classic symptomatology of ORN consists in intolerable pain, pathological fracture of compromised bone, devitalized bone sequestration, fistulous processes, which produce inability for patients to eat normally. The onset of ORN in less than two years after radiotherapy is due to the high doses of radiation of more than $70 \mathrm{~Gy}$ that received the patients affected by head and neck cancer. The development of ORN increased when clinicians had done concomitantly some surgical procedure after treatment with ionizing radiation.

Radiotherapy reduces cells proliferation from bone marrow, periosteal tissue and endothelial cells in the production of extracellular matrix components such as collagen type I, one of the most important constitutive proteins of bone tissue and root dentin [8]. Among the risk factors for the development of ORN, one of them considered the most important by the authors is ionizing radiation, whose different modalities to develop this complication include: the total dose, amount of energy per photon, brachytherapy, irradiation field and dose fractionation. Although new modalities of radiotherapy such as Modified Intensity Radiotherapy (IMRT) currently developed, it is remained cases of ORN. For this reason, further studies to clarify the pathogenesis

*Corresponding authors: Wilber Edison Bernaola Paredes, Nuclear and Energy Research Institute IPEN/CNEN Sao Paulo, Brazil, Tel: 5511981090791; E-mail: edison1488@hotmail.com

Received March 15, 2017; Accepted April 01, 2017; Published April 03, 2017

Citation: Paredes WEB, de Andrade DA (2017) ATR-FTIR Assessment of the Biochemical Composition and Micro Hardness of the Hard Tissues of Oral Cavity Submitted to Gamma Irradiation. J Cancer Sci Ther 9: 379-388. doi: 10.4172/19485956.1000446

Copyright: (C) 2017 Paredes WEB, et al. This is an open-access article distributed under the terms of the Creative Commons Attribution License, which permits unrestricted use, distribution, and reproduction in any medium, provided the original author and source are credited. 
of ORN, besides, the direct and isolated action of ionizing radiation on the hard tissues of the oral cavity must considered. Subsequently, clinicians could have established future modalities in the treatment and prevention of this deleterious effect of radiotherapy [4]. According to assessment [3], ORN is unlikely to appear when the radiation dose is less than $70 \mathrm{~Gy}$; but other study [4] refers to an increase in the incidence of ORN in patients who received doses between 65 and $70 \mathrm{~Gy}$, with an average dose of $66 \mathrm{~Gy}$. Currently, the high incidence (1\% to $30 \%)$ of the osteoradionecrosis $[3,4]$ that affects patients undergoing head and neck radiotherapy for the treatment of cancer, the present study aims to add a relevant data still unpublished in the literature.

Osteorradionecrosis is a complication that decreases the survival of irradiated patients, because of the risk of systemic and local infection, which considerably reduces them quality of life. Besides, osteoradionecrosis may require mutilating surgical treatment and therefore decreases mainly oral and physiological functions.

After the aforementioned, this assessment proposed to understand mechanism of development of this complication by the effect of ionizing radiation, involving all the hard tissues of the oral cavity, with the purpose of determining in vitro, the direct actions of the gamma radiation as an isolated risk factor for the development of undesirable effects.

Recent study [5] from the Biophotonics Laboratory of the Nuclear and Energy Research Institute-IPEN (São Paulo-Brazil) showed that, enamel submitted to ionizing radiation might not be more susceptible to demineralization than non-irradiated enamel, observed in vitro. For root dentin, with higher organic content than enamel, it was the most affected by gamma radiation compared to enamel ad coronary dentin, as demonstrated in the literature [6-8].

For irradiated patients, it is extremely important an oral environment adequate before radiotherapy treatment, besides proper nutrition and hygiene conditions maintained during and after radiotherapy. However, irradiated patients showed difficulties to deal with this routine, mainly due to the incidence of mucositis, muscle stiffness or trismus, and in the case of alveolar tissue, the presence of osteorradionecrosis.

Some studies [9-21] report unfavorable results in the tissues of oral cavity with greater organic content after radiotherapy treatment; however, it is necessary to analyze the amount of mineral loss that occurs in the hard tissues submitted to gamma irradiation [22].

Considering important to find preventive strategies in order to ensure and improve survival characteristics in the irradiated tissues and that these studies are impracticable to perform in vivo, because the ethical point of view, it must develop an in vitro protocol [14,15,23-27].

The determination of effects caused by ionizing radiation, using dose for patients with head and neck cancer, in terms of variations in biochemical composition and surface microhardness [22-31], as a first step as pathophysiology comprehension, will be helpful for reduced or to eliminate inherent radiotherapy complications. Moreover, it could focus on more details from specific issues as dose reduced fractionation protocols and modalities, as long as data obtained from in vitro studies [31-40].

This study evaluated physical properties, morphological aspects and composition of hard tissues in oral cavity submitted to gamma radiation under therapeutic dose. These parameters were determined by surface microhardness analysis; Scanning electronic microscopy (SEM) and attenuated total reflectance Fourier transform infrared spectroscopy (ATR-FTIR).

\section{Materials and Methods}

For the present study, we selected fifty healthy third human molars and two swine mandibles in order to obtain four groups submitted to analysis: enamel slabs ( $\mathrm{n}=50$ samples), root dentin slabs ( $\mathrm{n}=49$ samples), mandibular body slabs ( $\mathrm{n}=51$ samples), and retromolar region slabs ( $\mathrm{n}=49$ samples). It was approved for the ethical committee in human and animal for scientific studies of Energy and Nuclear Research Center, Sao Paulo, Brazil.

\section{Cutting and obtaining blocks of dental enamel and root dentin}

Fifty healthy human third molars selected from the bank's teeth from University of Sao Paulo, School of Dentistry (USP) were kept in thymol solution (concentration $1 \mathrm{~g} / \mathrm{L}$ ) for 48 hours and refrigeration at $4^{\circ} \mathrm{C}$. After the initial decontamination of the teeth, they were placed in a container with distilled and deionized water to begin the separation of the crowns and roots, in order to obtain slabs of dental enamel and root dentin.

A high-speed diamond-tipped instrument we used for making cuts at the level of the cemental enamel junction (CEJ). Afterwards, slabs were cleaned for removing remaining organic tissue, used curettes for scaling and root planning (Millenium $13 \mathrm{MC}$ and \# 34 straight, curve \# 28, Millenium \# 28 with fine point, São Paulo, Brazil). All slabs, each one submitted to ultrasonic cleaning (Unique Ultrasonic WasherThomton, Unique Ind. And. Com. Ltda., and São Paulo, Brazil) for a period of 30 minutes divided into two cycles of 15 minutes each one to eliminate organic debris removed.

In addition, slabs was placed in beakers with distilled and deionized water, positioned in the center of the washer tub, and after the first ultrasonic washing cycle, containers discharged and exchanged water for starting the second washing cycle. Finally, $20 \mathrm{ml}$ distilled water was poured and beakers were covered with aluminum foil and stored under refrigeration at $4^{\circ} \mathrm{C}$ for further macroscopic analysis.

For macroscopic analysis of slabs obtained, inclusion and exclusion criteria we established which included crowns and roots without visible superficial defects. Moreover, crowns and roots selected were fixed on acrylic sheet approximately $4 \mathrm{~cm}$ wide by $5 \mathrm{~cm}$ in diameter, using wax yellow sticky placed in a lamp and heated until it melted and was transported to the acrylic sheet by a metal dripper. A drop of wax positioned for the parallel positioning of one of the edges of the crown to the lateral edge of acrylic blade, and finally sides of the crown were fixed. Once the crown fixed to the blade, cut in a transversal and horizontal direction of manual cutter (IsoMet. Buehler, São Paulo, Brazil), with a fine-grained aluminum diamond disk (Struers, São Paulo, Brazil).

The crowns placed parallel to the largest arm of the equipment and disk directed for sectioning crown in a transversal way, with a distance approximately $3 \mathrm{~mm} \times 3 \mathrm{~mm}$ between cuts until reaching the basal part of the crown and finally obtained dental enamel samples with $3 \mathrm{~mm} \times$ $3 \mathrm{~mm} \times 1.2 \mathrm{~mm}$ as mean measures approximately.

On the other hand, slabs from root dentin were obtained as the same as dental enamel. The procedure carried out with previous fixation of the root and subdividing in three imaginary lines onto the frontal face in transversal and horizontal direction, for getting root dentin slabs of approximately $3 \mathrm{~mm} \times 3 \mathrm{~mm} \times 1.2 \mathrm{~mm}$. 


\section{Polishment protocol for enamel and root dentin samples}

Polishment of dental enamel slabs: Once the enamel and root dentin slabs obtained, we submitted to manual polishing in order to analyze the initial surface microhardness. Slabs mounted on the acrylic blade, fixed with sticky wax as previously described.

For the first group to get enamel samples, enamel surface fixed at first time onto acrylic blade in order to polish the remaining dentin and obtain a flat surface parallel to the edges of the blade used. Enamel slabs, polished in Politriz manual equipment, with grit discs of different grains: started with a 400 to 800 disc of coarse grain under refrigeration for 15 seconds with a turning speed of $100 \mathrm{rpm}$, then a 600 to 1200 disc under refrigeration for 10 seconds with a speed of $100 \mathrm{rpm}$. Thereafter, discs as slightly fine grain (2500) than others used before (were used for 10 seconds and at a speed of $100 \mathrm{rpm}$, and to finish a 4000 fine grain disc was used during the time and speed previously described.

Afterwards, polishment was completed with the ultra polishment using $1 \mu \mathrm{m}$ diamond solution (Buehler, São Paulo, Brazil) without refrigeration, poured into the felt disc and executed at a speed of 300 rpm for 1 minute. Therefore, this procedure described and performed for dental enamel surface, which was turned upwards and dentin polished was turned to the acrylic sheet, fixing it with sticky wax on the lateral edges with the precaution of avoiding contact between the wax and the block.

At the end of polishment slabs, samples obtained from dental enamel were submitted to ultrasonic washing for almost 40 minutes subdivided into 5 cycles of 8 minutes each one, and the distilled water was exchanged in each cycle. Beakers, included as samples into, with distilled and deionized water, were stored for 24 hours and subsequent fixation of the samples on the acrylic sheet.

Root dentin slabs, after manual polishment, as well as enamel, were submitted to ultra-polishment with $1 \mu \mathrm{m}$ diamond solution, without refrigeration, at a speed of $300 \mathrm{rpm}$ for 1 minute.

Finally, samples were submitted to ultrasonic washing, as described for enamel samples.

\section{Preparation of swine mandibular bone samples}

Swine jaws selected with measures $15 \mathrm{~cm}$ as length and $10 \mathrm{~cm}$ as width, provided by Raia Frigorific (Carapicuíba, São Paulo, Brazil). Jaws were washed with plenty of distilled and deionized water and then stored in sterile containers in freezer for maintenance of biological tissues not removed at a temperature of $-88^{\circ} \mathrm{C}$.

Remaining soft tissue removal was carried out in the Laboratory of preparation of biological samples of the Lasers and Applications Center, such as muscular fasciae, muscles and ligaments belonging to the tissue structure of the mandibular bone. It was used a scalpel sheet $\mathrm{n}^{\circ} 15$, a scalpel handle $\mathrm{n}^{\circ} 3$, and a toothless forceps for pressing the tissue and final excision (Figure 1). A thorough washing with distilled and deionized water was performed after complete removal of remaining tissues, obtaining a superficial clean and homogeneous. After surgical procedure, jaws were wrapped in a sealed plastic envelope and stored under a temperature of $-88^{\circ} \mathrm{C}$ for preservation of surgical pieces.

Surgical specimens were subdivided into two halves for better management of the hemimandibles on both sides (Figures 2 and 3). Slabs from mandibular body were obtained through manual cutting with a low rotation motor, with an aluminum diamond disc and a metallic carburundum ad with abundant irrigation with distilled water

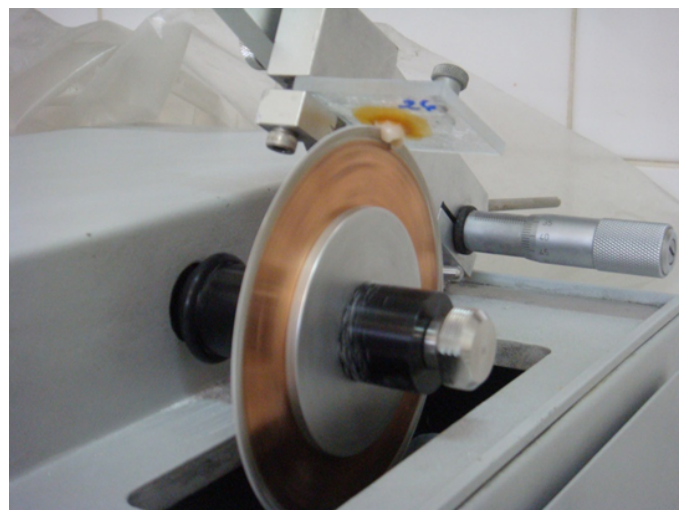

Figure 1: Cutting with fine-grained aluminum disc to obtain slabs from denta enamel.

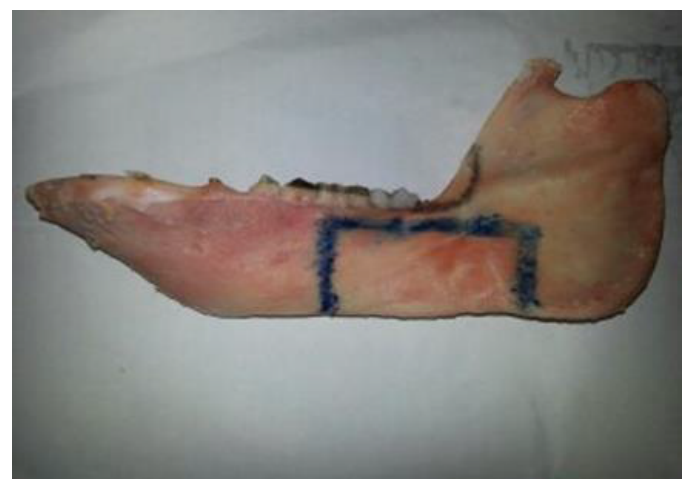

Figure 2: Delimitation of the medial region of mandibular body on the left side.

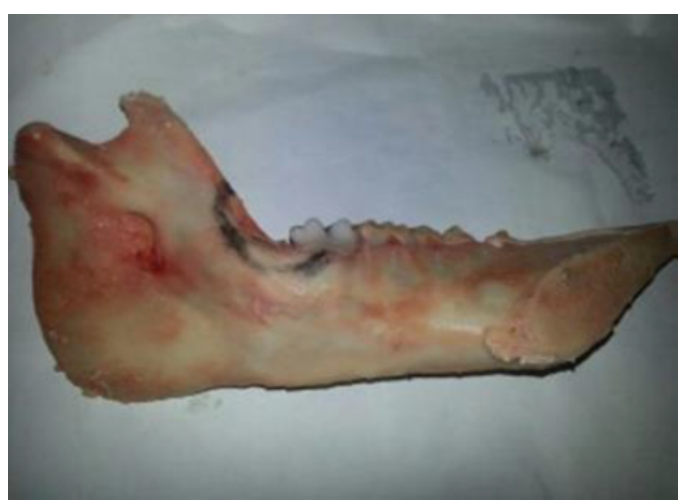

Figure 3: Delimitation of left trigone retromolar region.

in order to decrease the thermal effect after cutting. For retromolar region, the same procedure was done as well as mandibular body.

Afterwards, slabs submitted to ultrasonic washing to remove remaining organic tissues. Slabs, placed in sterile beakers with distilled and deionized water, followed by washing for 5 cycles of 8 minutes each one, and finally stored under a temperature of $-88^{\circ} \mathrm{C}$ until further analysis.

Mandibular body slabs were submitted to vertical and transversal cutting to obtain samples $3 \mathrm{~mm} \times 3 \mathrm{~mm} \times 1.2 \mathrm{~mm}$. They were fixed to the acrylic blade with sticky wax, with edges of the bone parallel to edges of the blade. Cutting started at the outermost edge of the block 
and continued to the inner edge, taking as a distance between cut and cut $3 \mathrm{~mm} \times 3 \mathrm{~mm} \times 1.2 \mathrm{~mm}$. Thereafter, samples were washed by ultrasonic washing for 15 minutes.

Afterwards, samples were fixed again to the acrylic sheet with sticky wax and the manual polishing of the same was carried out in politriz. Polishment protocol was done as well as described previously for enamel and dentin samples.

Therefore, ultrasonic washing was performed in order to remove remains of diamond solution. Samples were stored in a beaker with distilled and deionized water, covered hermetically and cooled to $4{ }^{\circ} \mathrm{C}$ for 24 hours.

For retromolar region preparation and polishment, samples were carried out at the same way as enamel, dentin, and body mandibular samples. Samples were fixed and submitted to initial surface microhardness analysis (baseline).

\section{Initial surface microhardness analysis (Baseline)}

Samples obtained and fixed on acrylic structure and submitted to initial surface microhardness (ISMH) analysis in order to obtain a quantitative assessment from samples surfaces after polishment.

Thus, for ISMH analysis (Baseline), a microdurometer (Shimadzu HMV-200, Japan) with Knoop indenter was used and programmed to apply a load of $245.2 \mathrm{mN}$ (HK 0.025) for 10 seconds for dental enamel samples, and a load of $98.4 \mathrm{mN}$ (HK 0.01) for 15 seconds (s) for root dentin, retro molar trigone region and mandibular body samples.

Each sample placed in microdurometer should be remained unmoved during throughout analysis. For each sample, 15 indentations were subdivided into three rows with 5 indentations each one, separated at distances of $100 \mu \mathrm{m}$ from a regular border, previously marked for future reference.

The distance between rows was $200 \mu \mathrm{m}$. After baseline indentations, the mean and standard deviation were calculated to verify homogeneous surface as purpose of inclusion criteria. Scatter plots were drawn in Microsoft Excel (Microsoft Office, 2013) for each group, leading to accurate selection of samples for been irradiated.

\section{Sample irradiation}

Samples submitted to gamma irradiation in the Cobalt-60 Irradiator (GammaCell, Co60) at the radiation technology center (CTR) of the Nuclear and Energy Research Institute (IPEN-CNEN/São Paulo, Brazil) using conventional irradiation protocol for patients with head and neck cancer.

Samples were placed in Petri plaques of resinous material for cultivation, and then submerged in cotton fields humidified with a solution of Thymol diluted in distilled water $(0.641 \mathrm{~g} / 1$ liter of distilled water) in order to maintain humid environment and to prevent fungi proliferation.

\section{Irradiation of dental enamel samples}

Dental enamel samples were placed in Petri's plaques submerged in cotton humidified with the Thymol solution and distilled water. These were distributed in 5 samples per Petri's plaques.

Then, irradiation protocol was initiated in the Cobalt- 60 Irradiator (Gamma Cell Source, Co60) with a dose rate of the equipment from March 2016 of $903 \mathrm{~Gy} / \mathrm{h}$, an intermediate dose of $0,76 \mathrm{~Gy}$, and finally a dose rate of $2 \mathrm{~Gy} / 4.94$ seconds. Irradiation was performed twice daily ( 4 Gy per day) at a 8 hours interval for each irradiation for 5 days until a weekly dose of 20 Gy was completed. After second irradiation, samples received irrigation with thymol solution, distilled water, and then stored under refrigeration at $4^{\circ} \mathrm{C}$.

\section{Irradiation of root dentine samples}

Root dentin samples submerged in humidified cotton with thymol and distilled water placed in Petri's plaques, ad submitted to gamma irradiation in the Cobalt-60 Irradiator (Gammacell Source, Co60) with conditions described before. Samples received a fractionated dose rate of 2 Gy/4.94 s twice daily, completing a dose of 20 Gy weekly. Total irradiation dose was $72 \mathrm{~Gy}$, and samples were rehydrated after the second irradiation time on the day.

\section{Irradiation of mandibular body samples}

Mandibular body samples, distributed in Petri' plaques in almost subgroups of 5 samples, were submerged in humidified cotton to maintain a humid environment and inhibited fungal growth. Immediately, samples were submitted to gamma irradiation as described above for others groups.

\section{Irradiation of retromolar trigone region}

For retromolar trigone samples, the same procedure as well as root dentin, dental enamel and mandibular body, was performed.

\section{Scanning electronic microscope analysis (SEM)}

Scanning electron microscopy (SEM) analyzed four samples for morphological assessment. At first, samples were treated and prepared (initial conditioning) physically for later analysis in SEM. Different concentrations of ethanol were chosen aimed for dehydrating each sample included on an increasing scale: $10 \%, 30 \%, 50 \%, 70 \%, 90 \%, 96 \%$ and $100 \%$ for 15 minutes in each solution.

Samples were examined in SEM TM 3000 (Tabletop Microscope) (Hitachi, Japan) from Materials Technology Center at IPEN/CNENSão Paulo, Brazil, with a voltage of $15 \mathrm{Kv}$ and Analy (EDS) for higher resolution of the image with a broad specificity of the surface to be analyzed. A conductive and double carbon adhesive tape was used to facilitate the fixing of the sample to the SEM sample port.

During analysis, it was obtained at least four images in different predetermined magnifications: first one analysis was developed with a magnification of $50 \mathrm{x}$, indicated for whole samples surfaces analysis, and, after that, other increaser magnifications of 500x, 1000x, 2000x, 4000x, were used.

Structural and morphological characteristics, we observed in each tissue after submitted to gamma irradiation, both superficial and regional effects mainly. Moreover, control group assessment (without irradiation) must been done in order to establish differences between initial and final group.

\section{Attenuated total reflection-Fourier transform infrared spectroscopy analysis (ATR-FTIR)}

The infrared spectra were acquired in a Fourier transform infrared spectrometer (FTIR-Perkin Elmer-model Spectrum 100 at IPEN/ CNEN, radiation technology center (CTR), São Paulo, Brazil), in ATR (attenuated total reflection) mode.

We assessed all samples in the middle infrared frequency range (from $4.000 \mathrm{~cm}^{-1}$ to $650 \mathrm{~cm}^{-1}$ ) was the adequate range to identify 
Citation: Paredes WEB, de Andrade DA (2017) ATR-FTIR Assessment of the Biochemical Composition and Micro Hardness of the Hard Tissues of Oral Cavity Submitted to Gamma Irradiation. J Cancer Sci Ther 9: 379-388. doi: 10.4172/1948-5956.1000446

all bands from organic and inorganic compounds present in nonirradiated and irradiated samples. The acquisition accuracy was $4 \mathrm{~cm}^{-1}$.

A greater number of scans used following the latest literature reports 32 completing 80 scans with background subtraction (BG). The crystal that made up the equipment for the analysis was the zinc selenide ( $\mathrm{ZnSe})$, in order to obtain a biochemical, qualitative and semi-quantitative analysis of the organic and inorganic composition. Samples were detached from the acrylic slides and positioned above the ZnSe crystal to obtain spectra generated by the Fourier transform. Immediately, after samples were centrally positioned on the crystal, a little adjustment against the crystal was followed using a specific tensile force $(130 \mathrm{~N})$ applied, which allowed an accurate analysis of the samples.

\section{Statistical Analysis}

Statistical package for Social Sciences, SPSS.18, used to determine the mean values of surface microhardness before and after irradiation. For this study, statistical t-student test was applied in order to compare differences between initial and final mean value of surface microhardness from each group. For analyzing the mean of differences between initial and final values from these four groups we applied ANOVA and Tukey test (inter- group differences) with a level confidence 95\% ( $\mathrm{p}<0.05 \%)$ (Figures 4-8).

\section{Results and Discussion}

\section{Scanning electronic microscope analysis (SEM)}

Images from samples before and after irradiation assessed to show morphological effects in and around surface. Details about undesirable's effects in mandibular body, caused by gamma irradiation

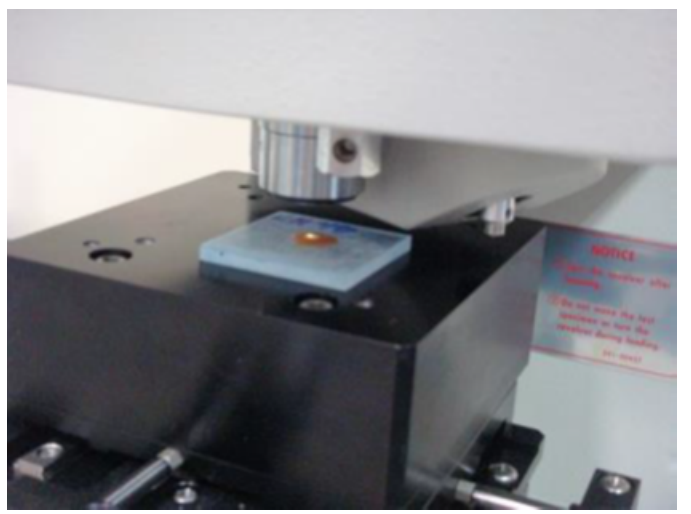

Figure 4: Initial surfaces micro hardness analysis with Knoop indenter.

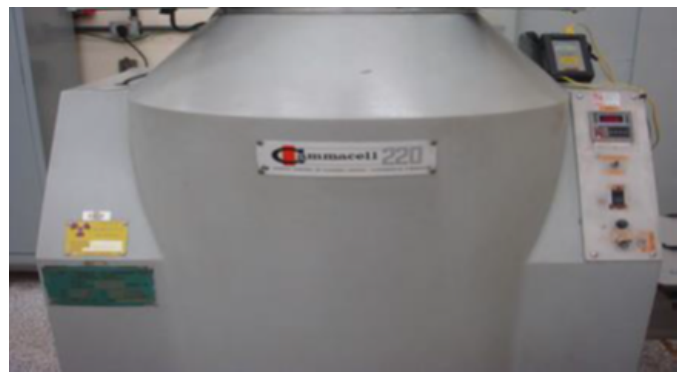

Figure 5: Cobalto-60 gamma cell irradiator source. Dose rate: 2 Gy/4, 4 seconds, received twice daily (total dose: $72 \mathrm{~Gy}$ ).

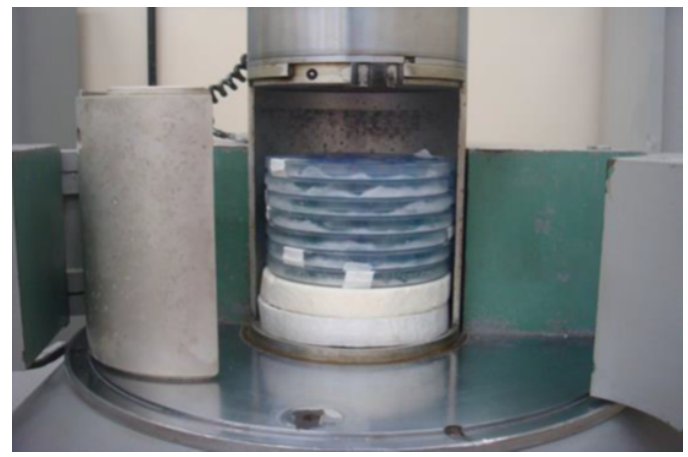

Figure 6: Placement of Petri's s plaques with different samples before submission to gamma irradiation.

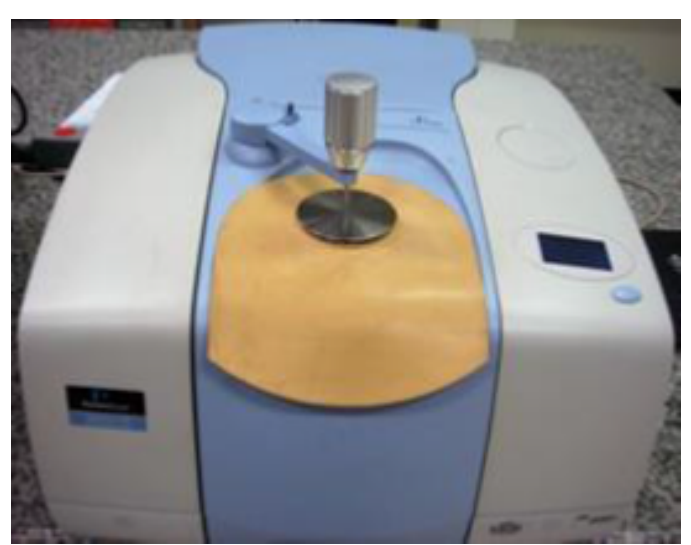

Figure 7: Fourier transformed by infrared spectroscopy analysis by spectrometer at CTR.

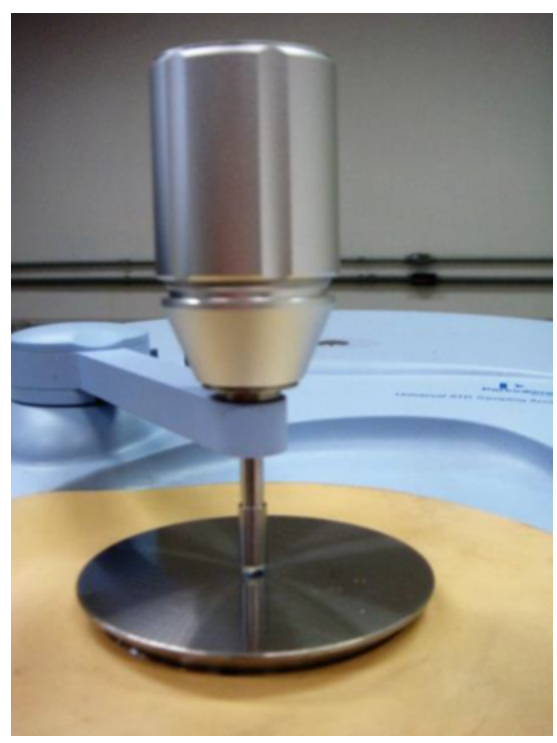

Figure 8: Attenuated total reflection analysis of samples with a tensile force of 130 .

on the surface of these hard tissues showed in Figure 9. Fractures, plenty of perforations and superficial cracks after samples submitted to irradiation, with possibilities for visualizing surface injure with 
Citation: Paredes WEB, de Andrade DA (2017) ATR-FTIR Assessment of the Biochemical Composition and Micro Hardness of the Hard Tissues of Oral Cavity Submitted to Gamma Irradiation. J Cancer Sci Ther 9: 379-388. doi: 10.4172/1948-5956.1000446

different magnifications, showing fragments tissue displacements, as well as loss of continuity of the tissue in relation to a surface smooth, flat and without bumpy or perforated areas. In Figure 9, there is progressive fragmentation around central nucleus, showed an especial structural disorganization, as comminuted fractures surfacing from mandibular body sample. In addition, variations of injures around surface, like a different types of cracks with less depth and smooth line fracture at central surface region. Image of retromolar region before gamma irradiation, after mechanical polishment, possible to identify a smooth and uniform surface corresponding to apparently healthy tissue at the macro-regional level is showed in Figure 10. In contrast, deleterious effects founded, with several details, is observed in the same image of the retromolar region sample after total dose of gamma radiation $(72$ Gy). The image shows a structure disorganization of central surface, presenting a considerable longitudinal crack, besides visualizing smaller cracks around the mean injure centered, whereby ionizing radiation effect at the irradiated site is greater deleterious for this location than other surface locations assessed. On the other hand, current and evident tissue instability created by gamma radiation leads to further destruction of this sample, with loss of surface hardness, meaningfully. Images from dental enamel samples before and after gamma irradiation were shown in the next figure. It is possible to observe a total structural disintegration and deterioration of the surface, justified with presence of plenty of cracks and fracture lines around central surface injured. In Figure 11, about magnification 2000x, it is possible to observe an extensive longitudinal crack including an irregular and bumpy shape, because of gamma radiation had been altered enamel's structure, besides for visualizing innumerous fragments of hard tissue involved. Finally, in Figure 12, we set out features from root dentin samples before and after gamma irradiation. At magnification of 2000x observed, after gamma irradiation, a line of fracture stands out, with slight displacement of edges and loss of surface continuity around this mineralized tissue, which again represented as a specific surface injure by ionizing radiation.

\section{Surface microhardness analysis (SMH)}

After surface microhardness analysis, before and after gamma irradiation, our results showed a considerable decrease in enamel and

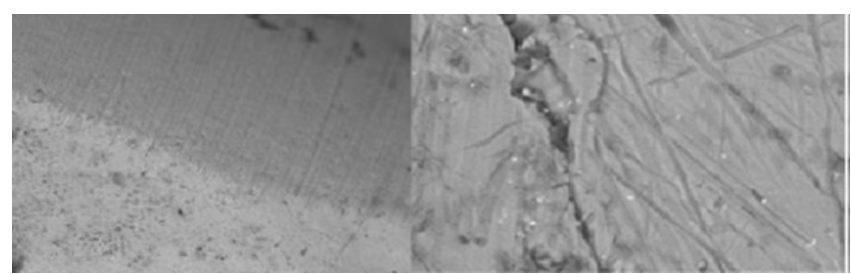

Figure 9: Mandibular body samples before and after gamma irradiation.

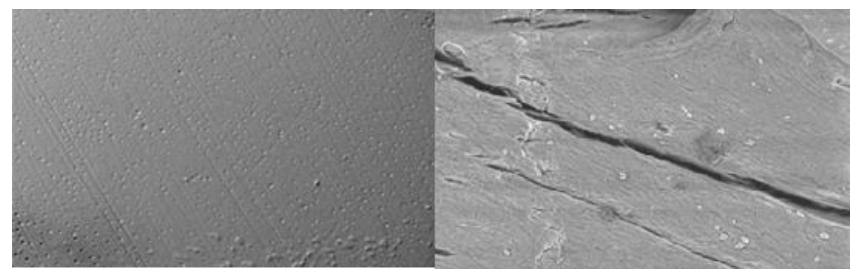

Figure 10: Trigone retromolar region samples before add after gamma irradiation.

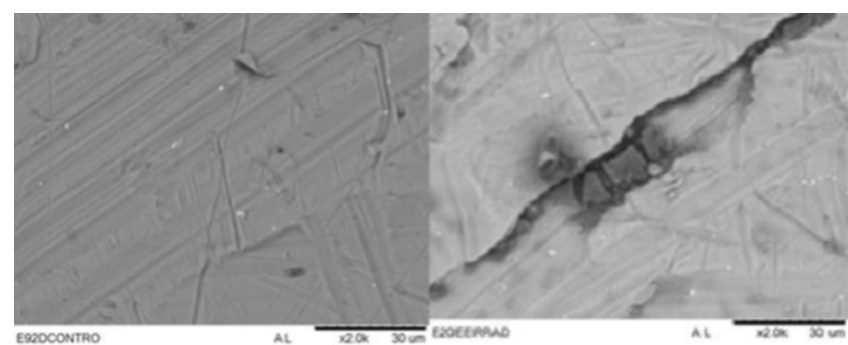

Figure 11: Enamel samples before and after gamma irradiation.

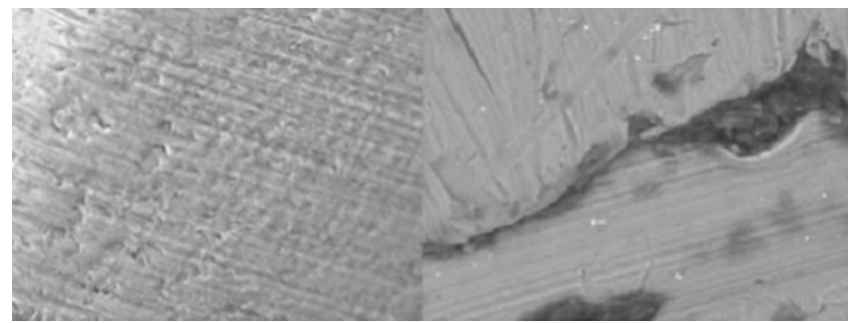

Figure 12: Root dentin samples before and after gamma irradiation.

root dentin hardness, corresponding to the results obtained by other studies [14,16-18].

Statistical analysis applied and normality, homogeneity and independence tests for results assessment was performed to establish the statistical models used. For initial statistical assessment, student's t-test of the related means was used for each group, with confidence level $95 \%(<0.05 \%)$, obtaining, in this study, a value of $\mathrm{p}=0.00$, contrasting the nullity hypothesis, supported that initial and final microhardness results, for each group, were statistically different. On the other hand, root dentin group showed a slight superiority results in terms of differences between initial and final microhardness values than other tissues with greater organic content, such as the mandibular body and retromolar trigone region.

Finally, mandibular body group and retromolar region showed results very close in loss of hardness when compared both mean results, therefore, it demonstrated that there were not statistically significant differences, as showed in Figure 13, supported that this results, reflecting the same effect by gamma irradiation in both tissues.

Enamel samples showed the highest mean value about difference between initial and final surface microhardness that others, groups of this study $(\mathrm{p}=0.00 \%)$. For this reason, it probably demonstrates statistically a greater injured enamel surface by gamma radiation, because of lower values from final microhardness obtained when compared with initial values as showed after application of ANOVA/ Tukey statistical test. Regarding root dentin, our study showed a lower mean value than obtained in enamel samples, but it was higher when compared to mandibular bone regions. According to the recent literature [14-16] dentin was the greatest hard tissue injured because of a major destruction by gamma radiation, considering as a mean factor, its organic composition, and water higher content than other hard tissues, besides other local factors like proteinases and metáloproteinases activated during the destructive and protein degradation process. 


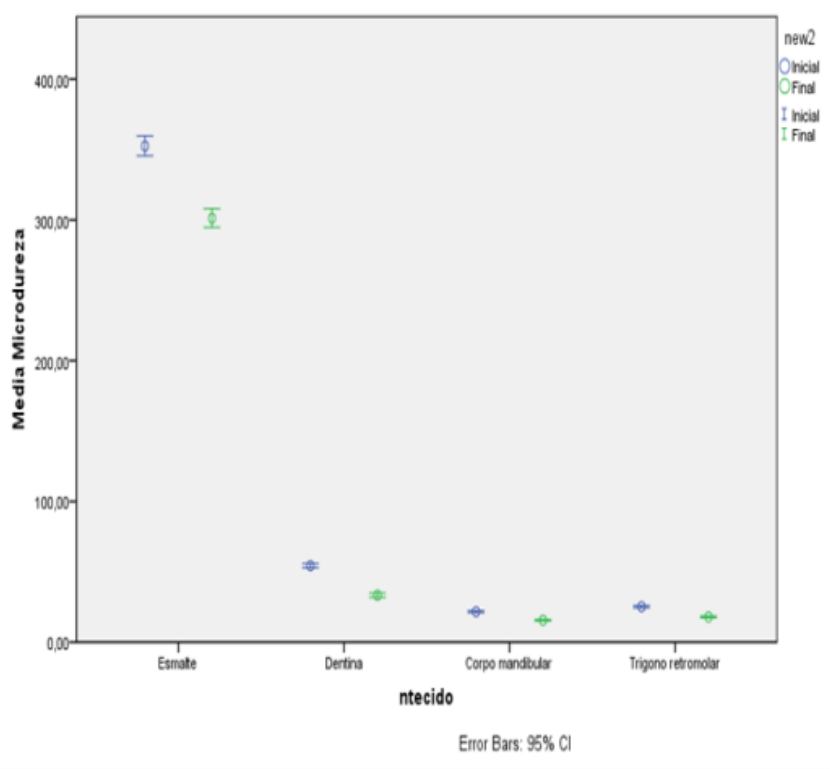

Figure 13: $T$ student test for analysis mean values of initial ad final micro hardness (level confidence: 95\%).

In this study, root dentin showed a lower rate injured than enamel, agreed with several previous studies [15-19] with a specific dose threshold (doses up to $30 \mathrm{~Gy}$ ), whose demonstrated greater hardness enamel loss than in higher doses. Nevertheless, results obtained in this study regarding enamel microhardness values would require further accurate assessment and consequently more studies will been carried out about direct and isolated effect of gamma radiation. Issues considerate for further studies since application modality, and specific deleterious effect on human teeth enamel, with consequent loss of hardness, caused, primarily, for a physic-chemical disorganization of mineralized tissues and subsequently a rapid advance in underlying tissues. Decay of radiation, characterized by rapid and aggressive advancement causes partial and total destruction of teeth in patients undergone to radiotherapy. In fact, it must require a definitely treatment, considerate as high complexity and difficult to manage, damaged thoroughly physical and psychologically these patients, justified due to deleterious effect on dental enamel, as demonstrated in this study (Figure 14).

Fourier transform infrared spectroscopy (FTIR) spectroscopy with the attenuated total reflection technique (ATR). The infrared spectra of enamel region are showed in Figure 15. The reference sample (nonirradiated-E69 REF) shows absorption bands at $3.425 \mathrm{~cm}^{-1}$, related to axial deformation of $\mathrm{O}-\mathrm{H}$ bond from hydroxyl group or $\mathrm{N}-\mathrm{H}$ bond from free amine group 45 ; at $2.916 \mathrm{~cm}^{-1}$ and $2.848 \mathrm{~cm}^{-1}$, related to asymmetric axial deformation of $\mathrm{C}-\mathrm{H}$ bond from methylene group of hydrocarbon chain; at $1.731 \mathrm{~cm}^{-1}$, related to axial deformation of $\mathrm{C}-\mathrm{O}-\mathrm{O}-$ bonds (peroxide group); at $1.465 \mathrm{~cm}^{-1}$ related to angular deformation from amide group (amide III) 46; at $1.165 \mathrm{~cm}^{-1}$, corresponds to asymmetric axial deformation of C-O-C bonds (ether group); at $1.034 \mathrm{~cm}^{-1}$ and $873 \mathrm{~cm}^{-1}$ related to infrared absorption of PO43- (phosphate group) and at $715 \mathrm{~cm}^{-1}$ corresponding to out-of-plane angular deformation of hydroxyl group linked by hydrogen bonds. This analysis suggests sample is formed by proteins, an inorganic region and water that is according to dental enamel composition, presented in the literature [41-44] specifically $97 \%$ of poly hydroxy apatite, $1 \%$ of protein, being ameloblastine, enamelin, amelogenin and $2 \%$ of water [45-47].

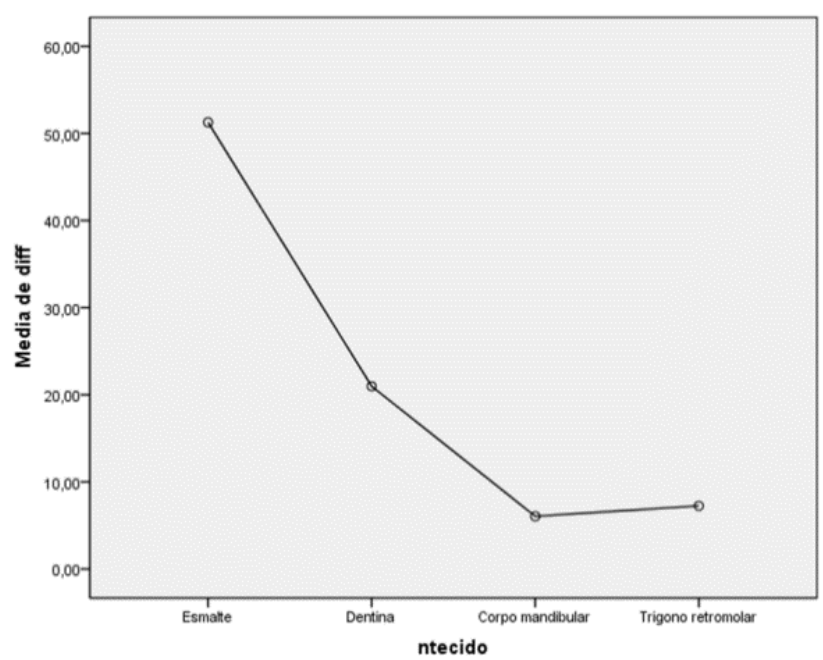

Figure 14: Mean difference between surface micro hardness results intergroups.

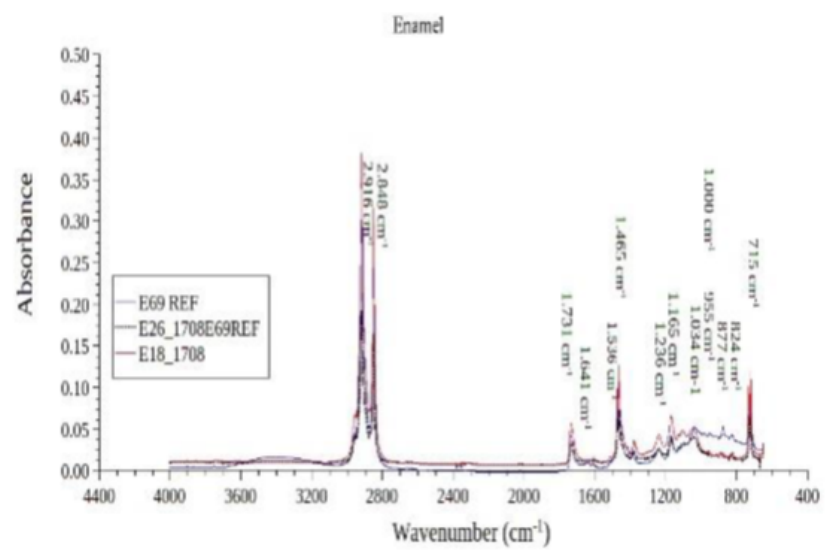

Figure 15: ATR-FTIR spectra from nonirradiated and irradiated enamel samples.

Irradiated samples (E26_1708 and E18_1708) showed almost the same absorption bands, however, the band at $3.425 \mathrm{~cm}^{-1}$ disappeared, suggesting water removal and a band at $877 \mathrm{~cm}^{-1}$ is observed, suggesting some changes in poly hydroxy apatite inorganic region (Figure 16) shows irradiated and non-irradiated root dentine spectra. Reference samples (non-irradiated) shows absorption bands at $3.283 \mathrm{~cm}^{-1}$, related to axial deformation of $\mathrm{O}-\mathrm{H}$ bond from hydroxyl group; at $3.085 \mathrm{~cm}^{-1}$ related to axial deformation of $\mathrm{C}-\mathrm{H}$ bond from hydrocarbon chain; at $1.630 \mathrm{~cm}^{-1}$ related to angular deformation from amide group (amide I) [48]; $1.544 \mathrm{~cm}^{-1}$ related to angular deformation from amide group (amide II) [48]; at $1.450 \mathrm{~cm}^{-1}$ and $1.401 \mathrm{~cm}^{-1}$ double peak related to angular deformation of $\mathrm{O}-\mathrm{H}$ bond from a carboxylic acid compound; at $1.338 \mathrm{~cm}^{-1}$ axial deformation of $\mathrm{C}-\mathrm{O}$ bond from a carboxylic acid; at $1.236 \mathrm{~cm}^{-1}$ related to amide group (amide III) [48]; at $993 \mathrm{~cm}^{-1}$ and $869 \mathrm{~cm}^{-1}$ related to infrared absorption of PO43- (phosphate group). This analysis suggests water is more important in this kind of sample than enamel's and amide group has more conformational modes that is compatible with root dentin composition: $70 \%$ inorganic material (poly hydroxy apatite and calcium phosphates), 18\% organic material 


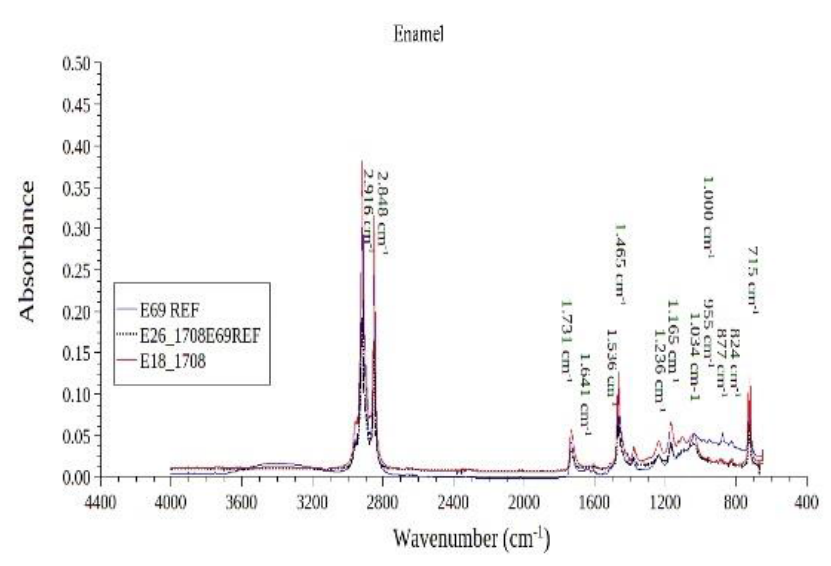

Figure 16: ATR-FTIR spectra from nonirradiated and irradiated root dentin samples.

(collagen type I, lipids, citrates, glycoproteins and proteoglycans) and $12 \%$ water. In irradiated samples, bands at $3.283 \mathrm{~cm}^{-1}$ disappear or present very low intensity suggesting water removal or water molecule scission; carbon chain break is suggested by band disappearing at 3.085 $\mathrm{cm}^{-1}$. Also, the absorbed dose applied in these samples (72 Gy), is responsible to promote protein degradation that is evidenced by absence of amine bands or very low intensity amine bands at $1.630 \mathrm{~cm}^{-1}, 1.544$ $\mathrm{cm}^{-1}$ and $1.236 \mathrm{~cm}^{-1}$; similar behavior occurs in carboxylic acid bands (at $1.450 \mathrm{~cm}^{-1} / 1.401 \mathrm{~cm}^{-1}$ and $1.338 \mathrm{~cm}^{-1}$ regions), that are evidence of proteins molecules. However, poly hydroxy apatite is evidenced by high intensity bands at $2.916 \mathrm{~cm}^{-1}$ and $2.848 \mathrm{~cm}^{-1}$ (organic phase support) and at $993 \mathrm{~cm}^{-1}$ and $869 \mathrm{~cm}^{-1}$ (sample inorganic phase). These results are still observed in Figure 17. These results show distinct behavior under radiation interaction in both enamel and root dentin samples. The peroxidation is a known process and occur in many class of chemical reactions, also it is triggered by water radiolysis. Radiation interaction with water produce scission of molecules, new reactive species and its recombination [47]; among these, peroxides are reactive and unstable compounds that improve radical chain reactions, where chemical degradation is a frequent process 48 . In this sense, the evaluation of peroxides in enamel sample is linked to chemical reactions in this exterior part of tooth independent of radiation process. Nonetheless, root dentin analysis reveal peroxides presence only in irradiated samples. In this case, this kind of sample has higher water concentration than enamel that leads water radiolysis when exposed to a gamma source. Mandibular body consists of $65 \%$ of inorganic material (polyhydroxy apatite and calcium phosphates), $25 \%$ of organic material (type I collagen fibers) and $10 \%$ of water, as well as dentin [42,44].

The spectra of mandibular body are shown in Figure 18. The references (non-irradiated CM50REF and CM58REF) show the same spectral bands achieved in root dentin references that suggest mandibular body have the same compositional characteristics than root dentin. These results converge to those presented on the literature, where mandibular body presents $65 \%$ of inorganic material (poly hydroxy apatite and calcium phosphates), $25 \%$ of organic material (type I collagen fibers) and 10\% of water [42]. Also, irradiated sample shows the same result observed to irradiated root dentin. These results confirm the same nature of these samples and the same behavior under ionizing irradiation, where the same discussion is made here.

However, the presence of the bands at $1.445 \mathrm{~cm}^{-1}$ related to the $-\mathrm{OH}$

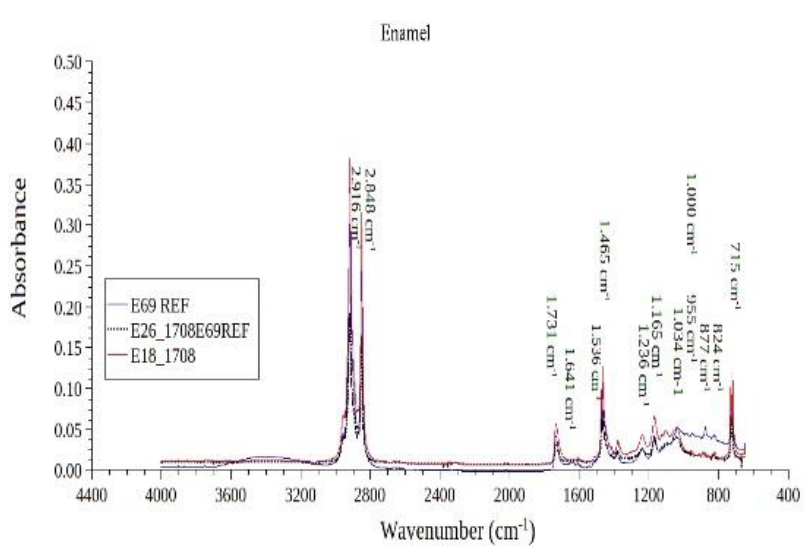

Figure 17: ATR-FTIR spectra from irradiated root dentin samples.

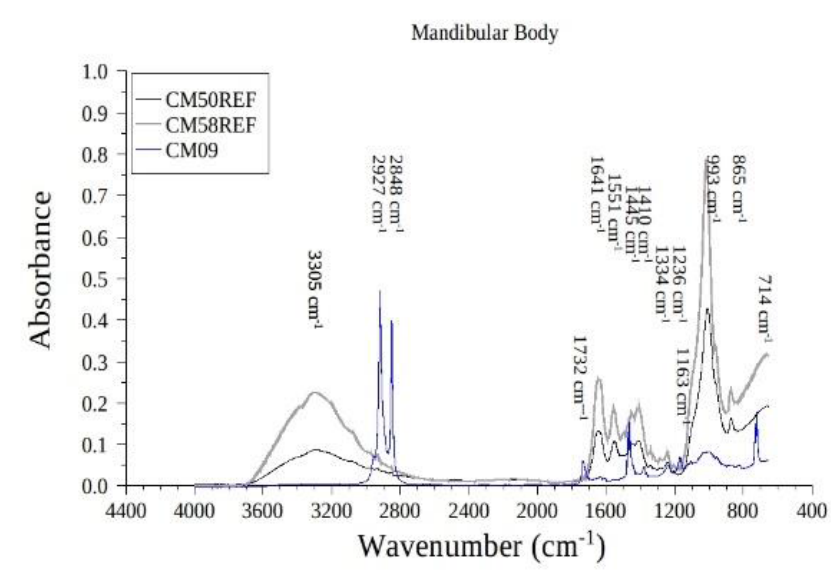

Figure 18: ATR-FTIR spectra from nonirradiated and irradiated mandibular body samples.

group of the carboxylic acid, $1.336 \mathrm{~cm}^{-1}$ related to the -CO group of the carboxylic acid and at $1.239 \mathrm{~cm}^{-1}$ related to the $-\mathrm{NH}$ (amide III) group are weak and less evident than in dentin.

Figure 18 compares the infrared spectral from non-irradiated samples (control group) and irradiated sample exposed to total dose of $72 \mathrm{~Gy}$, where it has been founded a great compositional alterations on spectrum for irradiated sample when compared to reference samples spectra. Because of bands disappeared in spectral regions at 3.291 $\mathrm{cm}^{-1}$, at $1.643 \mathrm{~cm}^{-1}$, at $1.553 \mathrm{~cm}^{-1}$, at $1.413 \mathrm{~cm}^{-1}$, at $1.012 \mathrm{~cm}^{-1}$ and at $865 \mathrm{~cm}^{-1}$. New bands appeared at $2.923 \mathrm{~cm}^{-1}, 2.852 \mathrm{~cm}^{-1}$, and regard to hydrocarbon chain from organic compounds, another band appeared at $1.745 \mathrm{~cm}^{-1}$ and at $1.734 \mathrm{~cm}^{-1}$ related to the $\mathrm{O}$-H-group bond from carboxylic acid. Besides, a peak band observed at $1.336 \mathrm{~cm}^{-1}$ region spectra regard to the - $\mathrm{CO}$ group of the carboxylic acid and finally at $715 \mathrm{~cm}^{-1}$, it corresponds to the out-of-plane angular deformation of $\mathrm{O}-\mathrm{H}$ group bonded by hydrogen bond from phosphate material. All compositional losses highlight a significant degradation happened in surface hard tissue, not only about organic compounds, but also about the breakdown of inorganic crystals, which relies upon oxidative action caused by presence of highly reactive substances and oxidants 
Citation: Paredes WEB, de Andrade DA (2017) ATR-FTIR Assessment of the Biochemical Composition and Micro Hardness of the Hard Tissues of Oral Cavity Submitted to Gamma Irradiation. J Cancer Sci Ther 9: 379-388. doi: 10.4172/1948-5956.1000446

such as peroxide. Finally, in retromolar trigone region, which has composition similar than mandibular body, but featuring a lower percentage of inorganic material (poly-hydroxyapatite and calcium phosphates) and a slight amount regarded to organic material (type I collagen fibers), besides, similar percentage of water. Non-irradiated samples of retromolar trigone region are shown in Figure 19. In these spectra, the same bands shown for root dentin and mandibular body are present; however we verified low intensity in bands at $993 \mathrm{~cm}^{-1}$ and at $869 \mathrm{~cm}^{-1}$ related to infrared absorption of PO43-(phosphate group). These results suggest chemical composition is similar also in retromolar trigone region, where the difference is a low phosphate concentration. These results are confirmed by literature [42]. Irradiated retromolar trigone region spectra (Figure 20), show the same bands of irradiated samples in Figures 16-18. However a difference is verified: some samples present high intensity bands at phosphate region, suggesting radiation degradation occur preferably in compositional water and proteins. No peroxide attributed band was observed. This may be related to an effective degradation process in distinct class of compounds that may be catalysed by peroxidation, even when the species is not evidenced in spectral results, (e.g. peroxide may be consumed in degradation process

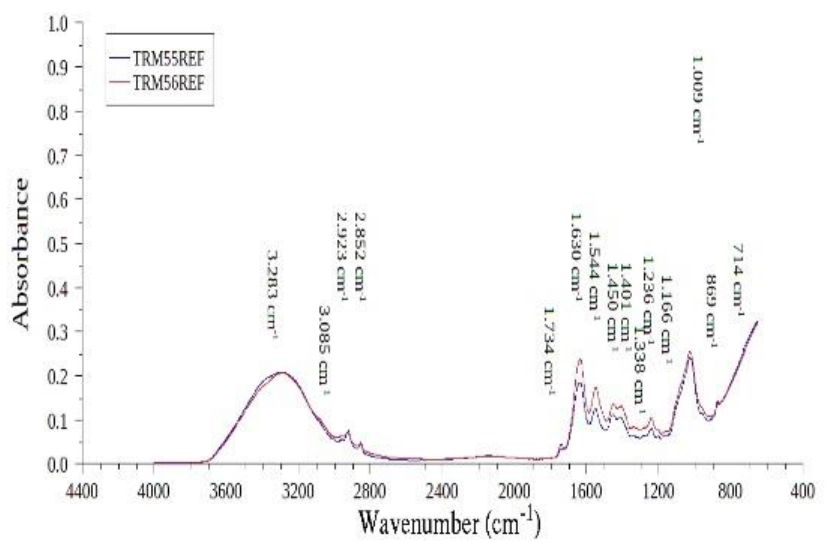

Figure 19: ATR-FTIR spectra from nonirradiated retromolar trigone region.

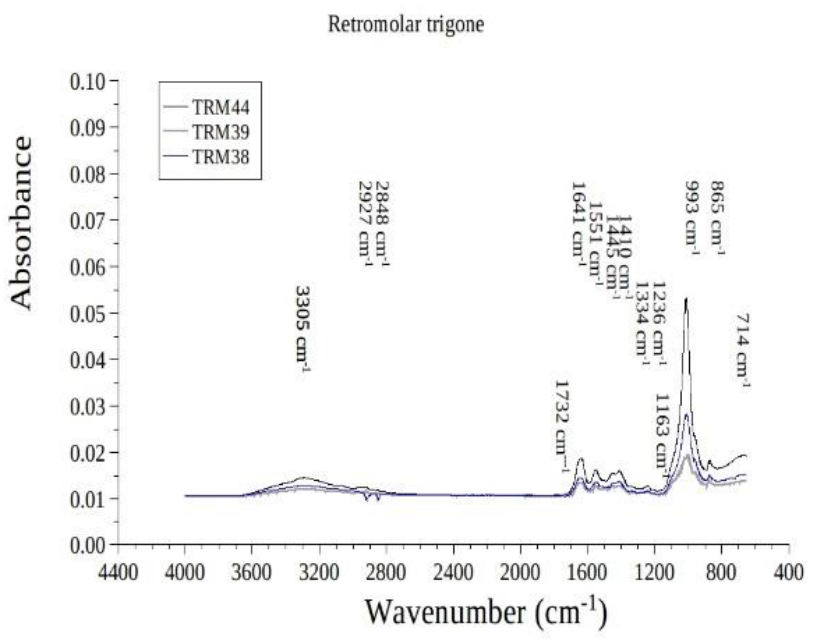

Figure 20: ATR-FTIR spectra from irradiated retromolar trigone region. or stay in very low concentration after process). In this case, inorganic compounds were saved in irradiation process.

\section{Conclusion}

After an accurate assessment and discussion about results, it was observed a high deleterious and statistical significant effect of ionizing radiation, due to loss of the physical, compositional and morphological properties on the hard tissues submitted to conventional radiotherapy, showed in this in vitro model.

Compositional infrared analysis of four groups submitted to irradiation process show distinct characteristics among them: root dentin and mandibular body present degradation in all compositional compounds and it is probably triggered by peroxides from water radiolysis; retromolar trigone region present high degradation of compositional water and proteins; inorganic compounds present low degradation and enamel regions suffer low changes in inorganic compounds and protein, but it was verified that water removal took place in this case. Dose rate used for treating patients affected by head and neck cancer, as conventional therapeutic modality, caused appearance of undesirable effects in hard oral tissues, independent of local or systemic contributing factors.

\section{References}

1. National Cancer Institute José Alencar Gomes da Silva. Ministry of Health (2015) Estimate incidence of cancer. Brazil (2016-2017).

2. National Cancer Institute José Alencar Gomes da Silva. Ministry of Health (2014) Estimate of incidence of cancer. Brazil (2014-2015)

3. Carini F, Bucalo C, Saggese V (2012) Case control study to assess the possibility of decrease the risk of osteoradionecrosis in relation to the dose of radiation absorbed by the jaw. Ann Stomatol 3: 3-7.

4. Grimaldi N, Sarmento V, Provedel L (2005) Dental care in prevention and treatment of osteoradionecrosis: Literature review. Revista Brasileira de Cancerologia 51: 319-354.

5. Zamataro CB, Ana PA, Benetti C, Zezell DM (2013) Influence of Er, Cr: YSGG laser on $\mathrm{CaF}_{2}$-like products formation because of professional acidulated fluoride or to domestic dentifrice application. Microsc Res Tech 76(7): 704-713.

6. McGuire JD, Mousa AA, Zhang BJ, Todoki LS (2014) Extracts of irradiated mature tooth crowns contains MMP-20 protein and activity. J Dent 42(5): 626-635.

7. Kadam SB, Shyama SK, Almeida VG (2013) Evaluation of the in vivo genotoxic effects of gamma radiation on the peripheral blood leukocytes of head and neck cancer patients undergoing radiotherapy. Mutant Res 752: 42-46.

8. Mellberg JR (1999) Hard tissue substrates for evaluation of cariogenic and anicariogenic activity in situ. Acta Odontol Scand 57(6): 334-341.

9. Arbabzadeh $F$ (2013) A Comparative study on microhardness and structural changes of dentin floor cavity prepared by Er: YAG Lasr irradiation and mechanical Bur. J Dent (Shiraz) 14(2): 73-74.

10. Koulourides T (1996) Dynamics of tooth surface-oral fluid equilibrium. Adv Ora Biol 2: 149-71

11. Kitajima EW, Leite B (1996) Introductory course on scanning electron microscopy, $2^{\circ}$ Edição. NAP/MEPA, Luiz de Queiroz College of Agriculture pp: 46.

12. Teruel Jde D, Alcolea A, Hernández A, Ruiz AJ (2015) Comparison of chemica composition of enamel and dentine in human, bovine, porcine and ovine teeth Arch Oral Biol 60: 768-775

13. Kunin AA, Evdokimova AY, Moiseeva NS (2015) Age-related differences of tooth enamel morphochemistry in health and dental caries. The EPMA J 6(3): $1-11$.

14. Reed R, Xu C, Liu Y (2015) Radiotherapy effect on nano-mechanical properties and chemical composition of enamel and dentine. Arch Oral Biol 60: 690-697.

15. Arsecularatne JA, Hoffman M (2014) An in vitro study of the microstructure, composition and nano indentation mechanical properties of remineralizing human dental enamel. J Phys D Appl Phys 1-13. 
Citation: Paredes WEB, de Andrade DA (2017) ATR-FTIR Assessment of the Biochemical Composition and Micro Hardness of the Hard Tissues of Oral Cavity Submitted to Gamma Irradiation. J Cancer Sci Ther 9: 379-388. doi: 10.4172/1948-5956.1000446

16. Gonçalves LM, Palma-Dibb RG, Paula-Silva FW (2014) Radiation therapy alters microhardness and microstructure of enamel and dentin of permanent human teeth. J Dent 42: 986-992.

17. Lieshout HF, Bots CP (2014) The effect of radiotherapy on dental hard tissue: A systematic review. Clin Oral Investig 18: 17-24

18. Ping Q, Huang S, Shanshan G (2015) Effect of gamma irradiation on the wear behavior of human tooth enamel. Nature, Sci Rep 5: 11568.

19. Deng J, Jackson L, Epstein JB (2015) Dental demineralization and caries in patients with head and neck cancer. Oral Oncol 51: 824-839.

20. Vissink A (2003) Oral sequelae of head and neck radiotherapy. Crit Rev Oral Biol Med 14(3): 199-212.

21. El-Faramawy N, Ameen R, El-Haddad K, El-Zainy M (2013) Effects of gamma radiation on hard dental tissues of albino rats: investigation by light microscopy. Radiat Environ Biophys 52(3): 375-387.

22. Trento CL (2006) Bone densitometry in the mandible of pigs submitted to autogenous, homogenous and heterogenous bone graft. Ph.D. Thesis, State University of São Paulo, School of Dentistry, Araçatuba.

23. Tymczyna B, Tatara MR, Krupski W (2012) Relationships between biochemical bone metabolism indices and morphometric, densitometric and mechanical properties of mandible in 6-month-old pigs. Ann Agric Environ Med 19(3): 535-539.

24. Li Y, Xu J, Mao L (2013) Allogenic mesenchymal stem cell therapy for bisphosphonate-related jaw osteonecrosis of swine. Stem Cells Dev (14): 2047-2056.

25. Brosh T, Rozitsky D, Geron S (2014) Tensile mechanical properties of swine cortical mandibular bone. PloS one 9(12): e113229.

26. Lin HS, Chen YJ, Li JD (2014) Measurement of mandibular growth using conebeam computed tomography: A miniature pig model study. PLOS ONE 9(5): $1-10$.

27. Pearce AI, Richards RG, Milz S (2007) Animal models for implant biomaterial research bone: A review 13: 1-10.

28. Hadley T, Song C, Wells L (2013) Does hyperbaric oxygen therapy have the potential to improve salivary gland function in irradiated head and neck cancer patients? Med Gas Res 3(15): 1-5.

29. Gevorgyan A, Wong K, Poon I (2013) Osteoradionecrosis of the mandible: A case series at a single institution. J Otolaryngol Head Neck Surg 42 (46): 1-7.

30. Niewald M, Fleckenstein J, Mang K (2013) Dental status, dental rehabilitation procedures, demographic and oncological data as potential risk factors for infected osteoradionecrosis of the lower jaw after radiotherapy for ora neoplasms: A retrospective evaluation. Radiat Oncol 8(1): 227.

31. Reuther T, Schuster T, Mende U (2003) Osteoradionecrosis of the jaws as side effect of radiotherapy of head and neck tumor patients-a report of a thirty year retrospective review. Int J Oral Maxillofac Surg 32(3): 289-295.
32. Freiberger JJ (2009) Multimodality surgical and hyperbaric management of mandibular osteoradionecrosis. Int J Radiat Oncol Biol Phys 75(3): 717-724

33. Lee IJ, Koom WS, Lee CG (2009) Risk factors ad dose-effects relationship for mandibular osteoradionecrosis. In oral and oropharyngeal cancer patients. Int J Radiat Oncol Biol Phys 75(4): 1084-1091

34. Bagan JV (2009) Osteonecrosis of the jaws by intravenous bisphosphonates and osteoradionecrosis: A comparative study. Med Oral Patol Oral Cir Bucal 14(12): e616-619.

35. Tamplen M (2011) standardized analysis of mandibular osteoradionecrosis in a rat model. Otolaryngol Head Neck Surg 145(3): 404-410.

36. Madrid C (2010) Osteoradionecrosis: A update. Oral Oncol 46: 471-474

37. Jegoux F (2011) Radiation effects on bone healing ad reconstruction: Interpretation of the literature. Oral Surg Oral Med Oral Pathol Oral Radio Endod 109(2): 173-184.

38. Zhuang Q (2011) Does radiation-induced fibrosis have an important role in pathophysiology of the osteoradionecrosis of jaw? Med Hypotheses 77: 63-65.

39. Curi MM (2016) Histopathologic and histomorphometric analysis of irradiation injury in bone and the surrounding soft tissues of the jaws. Surgical oncology and reconstruction. J Oral Maxillofac Surg 74(1): 190-199.

40. Curi MM (2007) Management of extensive osteoradionecrosis of the mandible with radical ad immediate microvascular reconstruction. J Oral Maxillofac Surg 65(3): 434-438.

41. Zezell DM (2015) FTIR spectroscopy revealing the effects of laser and ionizing radiation on biological hard tissues. $\mathrm{Br} \mathrm{J}$ Chem Soc 26: 2571-2582.

42. Figueiredo MM (2012) Characterization of bone ad bone-based graft materials using FTIR spectroscopy. Chemical engineering department, University of Coimbra Portugal.

43. Silverstein RM, Bessler GC, Morrill TC (1979) Spectrometric identification of organic compounds", (3rdedn.) Publisher Guanabara, Rio de Janeiro; p 127

44. Magne D, Weiss P, Bouler JM (2001) Study of the maturation of the organic (Type I Collagen) and mineral (Nonstoichiometric Apatite) constituents of a calcified tissue (dentin) as a function of location: A fourier transform infrared microspectroscopic investigation. J Bone Miner Res 16(4): 750-757.

45. Silverstein RM, Webster FX, Kiemle DJ, Bryce DL (2014) Spectrometric identification of organic compounds 8: 81-108.

46. Miyazawa T, Shimanouchi T, Mizushima SI (1958) Normal vibrations of N-Methyl acetamide. J Chem Phys. 29: 611-616.

47. Tabata Y (1991) CRC Handbook of radiation chemistry: CRC Press: 300-302. 48. Solomons TWG (2004) Organic chemistry 8: 447-449. 